\title{
Concepciones de la Felicidad y su relación con el Bienestar Subjetivo: un estudio mediante Redes Semánticas Naturales
}

\section{Conceptions of happiness and its relationship with subjective well-being: A study through Natural Semantic Networks}

\author{
Pablo Ezequiel Flores-Kanter ${ }^{\mathrm{a}, \mathrm{b}, *}$, Roger Muñoz-Navarroc, Leonardo Adrián Medrano ${ }^{\mathrm{b}}$ \\ aUniversidad Nacional de Córdoba, Argentina \\ bUniversidad Siglo 21, Argentina \\ 'Universidad de Valencia, España
}

\section{Resumen}

La búsqueda de la felicidad es uno de los fines más ansiados por los seres humanos. Así mismo, cada vez son más los estudios dentro de la psicología que buscan hallar cuáles son los factores involucrados en la felicidad. Existen dos grandes enfoques para su estudio, el paradigma hedonista y el paradigma eudaimónico. El paradigma hedónico propone que el bienestar depende principalmente de vivir una vida con experiencias placenteras; mientras que el paradigma eudaimónico refiere que el bienestar no solo se logra mediante el placer, sino principalmente a través de la actualización del potencial humano. En el presente estudio se trabajó con una muestra compuesta por 340 ciudadanos de Córdoba (Argentina) para indagar cuáles son sus concepciones sobre la felicidad mediante la técnica de Redes Semánticas Naturales (RSN). En este sentido, se propusieron dos hipótesis: 1) los participantes concebirán la felicidad tanto en términos hedónicos como eudaimónicos y 2) aquellos participantes con mayores niveles de felicidad tendrán una concepción predominantemente eudaimónica de la felicidad. La primera de las hipótesis pudo corroborarse de manera amplia dado que las definiciones obtenidas, tanto en la muestra total como en los grupos comparados, contenían tanto componentes hedónicos como eudaimónicos. Así mismo, se aporta evidencia a favor de la segunda hipótesis, esto es que los participantes con mayores niveles de felicidad subjetiva presentan una concepción predominantemente eudaimónica de la felicidad.

Palabras clave: felicidad, paradigma hedonista, paradigma eudaimónico, Redes Semánticas Naturales.

Para citar este artículo:

Flores-Kanter, P., Muñoz-Navarro, R., \& Medrano, L. A. (2018). Concepciones de la Felicidad y su relación con el Bienestar Subjetivo: un estudio mediante Redes Semánticas Naturales. Liberabit, 24(1), 115-130. doi: 10.24265/liberabit.2018.v24n1.08

\section{Abstract}

The pursuit of happiness is one of the most coveted goals of human beings. There are increasingly more psychology studies seeking to find which factors are involved in happiness. There are two main approaches to this study: the hedonic and the eudaemonic paradigms. The hedonic paradigm proposes that well-being depends primarily on living a life with pleasant experiences, while the eudaemonic paradigm refers that well-being is not only achieved through pleasure, but mainly through upgrading the human potential. In the present study we worked with a sample consisting of 340 citizens of Córdoba (Argentina) to investigate which conceptions they have about happiness by the technique of Natural Semantic Networks (NSN). Two hypotheses were proposed in this regard: 1) participants will conceive happiness both in hedonic and eudaemonic terms, and 2) those participants with higher levels of happiness will have a predominantly eudaemonic conception of happiness. The first hypothesis was corroborated broadly, because the definitions obtained in the total sample and in the groups being compared contained both hedonic and eudaemonic components. Likewise, evidence favoring the second hypothesis is also provided; that is, participants with higher levels of subjective happiness have a predominantly eudaemonic conception of happiness.

Keywords: happiness, hedonic paradigm, eudaemonic paradigm, Natural Semantic Networks.

Este es un artículo Open Access bajo la licencia Creative Commons Atribución-NoComercial-CompartirIgual 4.0

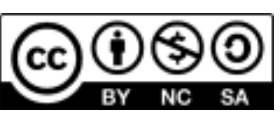




\section{Introducción}

La búsqueda de la felicidad y el logro del bienestar (desarrollo del potencial humano o de la buena vida) son uno de los fines humanos que se buscan desde la antigüedad (Boehm \& Lyubomirsky, 2009; Lyubomirsky \& Lepper, 1999; Riff \& Singer, 2007). Asimismo, cada vez son más los estudios dentro de la ciencia psicológica que buscan hallar cuáles son los factores involucrados en el bienestar y la salud (Aspinwall \& Staudinger, 2007). Diversas variables han sido estudiadas en este sentido y se han propuesto diversos factores asociados al potencial humano, como es la sabiduría (Baltes \& Freund, 2007), el afecto o las emociones positivas (Cohn \& Fredrickson, 2009; Isen, 2007), las creencias de autoeficacia (Maddux, 2009), y la creatividad y las experiencias de flow (Averill, 2009; Nakamura \& Csikszentmihalyi, 2007). Como indican Nakamura y Csikszentmihalyi (2007), estos paradigmas emergentes enriquecen a la psicología, la cual había seguido tradicionalmente un paradigma más patológico de la psiquis humana.

La presente investigación sigue esta línea de identificar factores asociados a una experiencia subjetiva de mayor felicidad. Es importante mencionar que no se realizará una distinción entre los términos felicidad y bienestar, sino que serán tomados como sinónimos, dado que si bien el término bienestar ha sido implementado en mayor medida en la literatura, por lo general los investigadores los mencionan intercambiablemente (Boehm \& Lyubomirsky, 2009; Caunt, Franklin, Brodaty, \& Brodaty, 2013; Lyubomirsky \& Lepper, 1999). Así mismo, el término felicidad ha sido más utilizado generalmente desde la perspectiva hedónica (Diener, Oishi, \& Lucas, 2009; Ryan \& Deci, 2001) para referenciar una experiencia subjetiva-afectiva predominantemente positiva o placentera. En cambio, se hará un uso más generalizado y se podrá observar que el término felicidad puede ser abordado desde diferentes perspectivas (Caunt et al., 2013; López-Pérez, Sánchez, \& Gummerum, 2015).
De este modo, resulta relevante observar los paradigmas a través de los cuales se ha definido la felicidad o el bienestar. En términos generales, el concepto de bienestar refiere a una experiencia y funcionamiento psicológico óptimo. Sin embargo, como plantean Ryan y Deci (2001), existe un gran debate respecto a lo que se considera una experiencia óptima y qué constituye una buena vida. Además, estos autores plantean la tesis de que es fundamental conocer cuáles son las acciones que las personas implementarán justamente para lograr el bienestar o felicidad, especificando que la forma en que uno defina el bienestar impactará en las actividades que se lleven a cabo en los diversos ámbitos de la vida.

Volviendo a los paradigmas que definen el bienestar o la felicidad, los mismos pueden sintetizarse en dos grandes enfoques (Ryan \& Deci, 2001): el paradigma hedonista y el paradigma eudaimónico. El paradigma hedónico propondrá que el bienestar depende principalmente de vivir una vida con experiencias predominantemente placenteras; mientras que el paradigma eudaimónico referirá que el bienestar no solo se logra mediante el placer, sino principalmente a través de la actualización del potencial humano. Ejemplos actuales del primer paradigma, el hedónico, lo encontramos actualmente desarrollado en la teoría del bienestar subjetivo (Diener et al., 2009), que define al bienestar como resultado de la evaluación cognitiva y afectiva que una persona haga acerca de su vida como un todo. De esta forma, el bienestar subjetivo se logra mediante la experimentación de niveles altos de afecto positivo, la vivencia de niveles bajos de afecto negativo y una alta satisfacción vital. Por otra parte, el segundo paradigma, la visión eudaimónica, queda representado en la teoría del bienestar psicológico (Riff \& Singer, 2007), desde donde se propone que el bienestar se alcanza a través de la consecución de los siguientes rasgos positivos: el propósito en la vida, el dominio del medio ambiente, las relaciones positivas, la autoaceptación, el crecimiento personal y la autonomía. Es decir, la felicidad puede entenderse desde un modelo hedónico o, en cambio, desde una visión eudaimónica, 
entendida como procedente del anhelo por superar los desafíos de la trayectoria vital mediante los rasgos del potencial humano.

Como se mencionó anteriormente, la forma en que se defina la felicidad o el bienestar no es un punto menor. Diversos estudios se han llevado a cabo para verificar si las diversas concepciones de felicidad que tengan las personas (hedónica vs. eudaimónica) se asociana los niveles de bienestar percibidos por las mismas. La concepción de felicidad puede entenderse como una representación cognitiva individual de la naturaleza y experiencia del bienestar (Bojanowska \& Zalewska, 2015). Estas concepciones generalmente pueden describirse como el grado en que las personas enfatizan dimensiones hedónicas o eudaimónicas como aspectos importantes para la experiencia del bienestar (McMahan \& Estes, 2011). Solo recientemente las investigaciones sobre el bienestar han comenzado a dar relevancia a este aspecto, es decir, al reconocimiento de la importancia de diferenciar entre perspectivas hedónicas y eudaimónicas de la felicidad (McMahan \& Renken, 2011).

En esta línea, Bojanowska y Zalewska (2015) indagaron si algunas concepciones de felicidad eran más beneficiosas que otras en términos de experiencia de bienestar subjetivo. Si bien indican que la satisfacción vital fue menor si la felicidad se asociaba con posesiones materiales, mientras que aumentaba si la felicidad se asociaba con las relaciones interpersonales, las correlaciones evidenciadas fueron de muy baja magnitud ( $r$ entre .07 y .08), por lo cual es criticable la significancia teórica-práctica de este estudio más allá de la significación estadística de dichas correlaciones. Algo similar puede plantearse para la correlación evidenciada en el mismo trabajo entre la concepción de la felicidad asociada a la experiencia del dominio en el trabajo y el afecto positivo $(r=.12)$, aunque aquí la proporción de varianza explicada sería de al menos el 1\%. Por su parte, McMahan y Estes (2012), utilizando la Escala sobre Creencias del Bienestar (BWBS), se propusieron como objetivo indagar qué creencias sobre el bienestar estaban más asociadas al mismo, comparando dos grupos de participantes, jóvenes y adultos. En los jóvenes se observó que las creencias de autodesarrollo presentan una correlación positiva con el afecto positivo $(r=$ .23), la experiencia subjetiva de felicidad $(r=.20)$, la vitalidad $(r=.36)$, y la autoestima $(r=.19)$. Para el caso de los adultos se observan algunas asociaciones adicionales y, a su vez, correlaciones más intensas. Así, las creencias relacionadas con las experiencias de placer se correlacionan de manera positiva con al afecto positivo $(r=.44)$, la experiencia subjetiva de felicidad ( $r=.45)$, el significado de la vida $(r=.42)$, y la vitalidad ( $r=.46)$. Por su parte, en las creencias relacionadas con la evitación de experiencias negativas, se observan también correlaciones positivas con el afecto positivo ( $r=.25$ ), el significado de la vida ( $r=.33)$, y la autoestima $(r=.32)$. Finalmente, en relación a las creencias de autodesarrollo se observan correlaciones positivas con las variables del afecto positivo $(r=.49)$, la experiencia subjetiva de felicidad $(r=.41)$, el significado de la vida $(r=.46)$, la vitalidad $(r=.43)$, y la autoestima $(r=.41)$.

Sumadas a las anteriores investigaciones, McMahan y Renken (2011) propusieron medir la relación entre las concepciones eudaimónicas del bienestar (e.g., creencias de autodesarrollo) con autoreportes de bienestar. Además, plantearon como hipótesis que el significado de la vida mediaría la relación indicada anteriormente. Los resultados permiten apreciar por un lado que las concepciones eudaimónicas del bienestar (e.g., creencias de autodesarrollo y contribución a los otros) se asocian positivamente $(\beta=.47, p<.01)$ con la experiencia de bienestar (e.g., satisfacción con la vida, vitalidad, afecto positivo y bajo afecto negativo). En este caso, al analizar una relación directa entre las variables, puede verse que las concepciones eudaimónicas del bienestar explicarían un $17 \%$ de la experiencia de bienestar. Posteriores análisis indicaron que el significado de la vida modera parcialmente la relación entre las concepciones eudaimónicas del bienestar y la experiencia de bienestar. Se observan así 
asociaciones positivas entre las concepciones eudaimónicas del bienestar y el significado de la vida $(\beta=.37, p<.01)$, y entre el significado de la vida y la experiencia de bienestar $(\beta=.71, p<.01)$; mientras que la asociación directa entre las concepciones eudaimónicas del bienestar con la experiencia de bienestar se vuelve menos intensa en este modelo aunque aún significativa ( $\beta=.15, p<$ .05). Lo interesante en este punto es que este modelo permite explicar un $60 \%$ de la experiencia del bienestar.

De manera similar a los anteriores hallazgos, McMahan y Estes (2011) verificaron que las creencias eudaimónicas presentaban asociaciones más intensas con el bienestar en comparación a las creencias hedónicas. Más específicamente, las creencias hedónicas mostraron una correlación positiva de baja intensidad con las variables bienestar subjetivo total ( $r=.21)$ y vitalidad $(r=.23)$; mientras que las correlaciones con las creencias eudaimónicas fueron positivas y moderadas, en relación al bienestar subjetivo total ( $r=.30)$, la vitalidad $(r=.34)$ y el significado de vida $(r=$.33). Posteriormente, al aplicar un análisis de regresión jerárquica, pudo observarse que las creencias hedónicas no permiten predecir de forma significativa las dimensiones del bienestar cuando se controla por las asociaciones entre las creencias eudaimónicas y dichas dimensiones; en cambio, las creencias eudaimónicas sí presentan predicciones significativas con el bienestar subjetivo total $(\beta=.28, p<.01)$, el significado de vida $(\beta=$ $.37, p<.01)$ y la vitalidad $(\beta=.32, p<.01)$, al controlar las asociaciones por las creencias hedónicas.

Siguiendo las líneas de investigaciones desarrolladas, la presente investigación tiene como principal objetivo indagar si el modo de concebir la felicidad se relaciona con un mayor o menor nivel de felicidad percibido; en otras palabras ¿las personas más felices tienen un modo más hedónico o más eudaimónico de concebir la felicidad? A partir de los antecedentes en el tema, el presente estudio plantea las siguientes hipótesis:
1. los participantes concebirán la felicidad tanto en términos hedónicos como eudaimónicos; sin embargo,

2. aquellos participantes con mayores niveles de felicidad tendrán una concepción predominantemente eudaimónica de la felicidad.

Para dar respuesta a estos interrogantes se utilizará una técnica que ha demostrado utilidad en la indagación de las concepciones o significados que las personas otorgan a un determinado constructo, y que no ha sido utilizada en los estudios previos sobre la temática: la Técnica de Redes Semánticas Naturales, que será explicada en mayor detalle en el apartado de procedimiento. Si bien Denegri, García y González (2015) utilizaron una técnica similar para indagar las definiciones dadas del bienestar, la estrategia de análisis implementada no posibilita la visualización de la red semántica en un grafo. Esto es, poder visualizar los conceptos que definen a un determinado constructo y cómo estos conceptos se relacionan entre sí para otorgar significado. Esta posibilidad, como se verá más adelante, no tiene que ver solo con una manera diferente de mostrar los mismos datos, sino que, por el contrario, permite una comprensión más acabada de la interrelación de los conceptos entre sí que no es posible apreciar con la técnica de análisis aplicada por los autores mencionados.

Finalmente, como plantean Bojanowska y Zalewska (2015), recientemente se ha empezado a tener interés en esta temática, por lo que resulta crucial seguir llevando a cabo investigaciones en esta área de investigación. En efecto, se han llevado a cabo hasta el momento escasos estudios con objeto de indagar las concepciones de la felicidad y su relación con el bienestar. Sumado a lo anterior, no existe hasta el momento evidencia de esta relación en la población Argentina. Todo lo anterior da cuenta de la relevancia de la presente investigación. 


\section{Metodología}

\section{Participantes}

En el presente estudio se trabajó con una muestra compuesta por 340 ciudadanos de Córdoba, Argentina, seleccionados a través de un muestreo no probabilístico accidental (Bologna, 2012). Dado que se trata de un muestreo no probabilístico, se buscó que la muestra sea lo más heterogénea en términos de edad, profesión y sexo. En cuanto a la edad, las mismas estuvieron comprendidas entre los 18 y los 66 años $(M=31.05 ; D E=12.47)$. Respecto al sexo, del total de participantes, el 27.1\% $(f=92)$ corresponde al sexo masculino, mientras que el $72.9 \%$ restante $(f=248)$ al sexo femenino. Finalmente, el $.9 \%(f=3)$ había alcanzado como máximo el nivel primario de estudios; el 19.1\% ( $f=65$ ), el secundario; el $17.4 \%(f=59)$, el terciario; $y$ el $62.6 \%(f=213)$, el nivel universitario.

\section{Instrumentos}

En primer lugar, para la indagación de las concepciones sobre la felicidad se utilizó una técnica denominada Redes Semánticas Naturales (RSN). Dado que cuando se habla de las concepciones de la felicidad se está haciendo referencia a una representación cognitiva individual de la naturaleza y experiencia del bienestar (Bojanowska \& Zalewska, 2015), esta técnica resulta especialmente útil. En este sentido, la misma permite conocer el significado que los sujetos tienen sobre un concepto dado (Valdez, 2002). En este caso, se utilizó para observar cuál es la concepción que los participantes manifestaban acerca de la felicidad. Esta técnica consiste en pedirle a los sujetos que definan el estímulo o reactivo (i.e. felicidad) con un mínimo de cinco palabras sueltas (Ramírez, Martínez, Montemayor, \& Nieto, 2009).

Por su parte, la Escala de Felicidad Subjetiva, desarrollada por Lyubomirsky y Lepper (1999), se aplicó con el fin de conocer el nivel de felicidad subjetiva experimentada por los participantes, dado que esta escala permite obtener una medida general de la felicidad. La misma consta de 4 ítems con respuesta tipo Likert con 7 opciones de respuesta (e.g., algunas personas en general son muy felices y disfrutan la vida pase lo que pase, aprovechándola al máximo. ¿Hasta qué punto esta descripción es válida para ti?). Es importante mencionar que la escala cuenta con estudios psicométricos adaptados a la población de Argentina (Medrano, 2013), que sugieren que la escala presenta una estructura factorial unidimensional, elevada consistencia interna y evidencias favorables de validez convergente.

\section{Procedimiento}

Para la consecución de los objetivos propuestos se utilizó un diseño de tipo ex post facto retrospectivo con dos grupos, uno de cuasi control (Montero \& León, 2006).

Respecto al procedimiento para la recolección de los datos, en primer lugar todos los participantes fueron informados sobre los objetivos de la investigación, y sobre su participación voluntaria y anónima en la misma. Tras obtener el consentimiento de los participantes, se aplicaron los distintos instrumentos de evaluación. Primero se administró a los estudiantes la Escala de Felicidad Percibida y, posteriormente, se aplicó la técnica RSN.

Finalmente, el análisis de los datos consistió en los siguientes procedimientos. Para analizar las hipótesis propuestas se decidió, en primer lugar, trabajar con la muestra total con el objeto de conocer cómo se definía la felicidad en todoslos participantes. Luego, a los fines de la comparación, fue preciso trabajar con dos grupos extremos: el 25\% del total de participantes que manifestaran mayor nivel de felicidad (e.g., GFA) y el 25\% que manifestara un nivel más bajo de felicidad (e.g., GFB); estos grupos quedaron determinados por los percentiles 75 y 25 respectivamente. Respecto al análisis propiamente dicho, antes de comparar a los grupos extremos con respecto a las concepciones de felicidad, se calculó la prueba $t$ para muestras independientes, para verificar si los grupos 
presentaban diferencias en cuanto al nivel de felicidad experimentado. De manera complementaria, se calculó la $d$ de Cohen para conocer el tamaño del efecto de las diferencias en los niveles de felicidad (Thalheimer \& Cook, 2002). Para los análisis descritos hasta aquí se utilizó el software IBM SPSS 20.

Por otra parte, el análisis de redes semánticas consistió principalmente en (1) el cálculo de indicadores de centralidad, y en (2) la disposición de la red en un diagrama para poder ser visualizada. El indicador de centralidad utilizado fue la centralidad de grado con pesos, el cual se calcula a partir del número de lazos o conexiones que tiene un nodo determinado con otros nodos más la coocurrencia entre dos nodos particulares. Este indicador permite identificar la importancia que tiene un nodo en una red determinada (Borgatti, Everett, \& Johnson, 2013), en otras palabras, permite detectar qué conceptos son los más centrales o importantes en la definición de la felicidad en la muestra total y en cada uno de los grupos contrastados. Finalmente, se decidió considerar las 15 palabras definidoras con mayores valores de centralidad como las más representativas en la concepción de la felicidad (Valdez, 2002). Obtenido el conjunto de 15 palabras, las mismas luego fueron agrupadas según el paradigma que más les correspondiera.

Esta categorización posterior se hizo siguiendo las definiciones dadas por Ryan y Deci (2001) acerca del enfoque hedonista y eudaimónico. Por otra parte, para el trazado o disposición de la red semántica en un gráfico se aplicó el algoritmo ForceAtlas 2 (Jacomy, Heymann, Venturini, \& Bastian, 2011). Este algoritmo utiliza un sistema de trazado de fuerzas dirigidas, esto es, simula un sistema físico: los nodos se repulsan entre sí (como los imanes) mientras que las aristas atraen los nodos que estas conectan (como un elástico o resorte). Sumado a lo anterior, el trazado de grafos de fuerzas dirigidas se caracteriza por ubicar los nodos en función de su relación con los otros nodos que componen la red, y no en función de las características contingentes propias que tenga un nodo en sí. Para este conjunto de análisis mencionado se utilizó el software Gephi 0.8.

Por último, con la finalidad de obtener no solo valores descriptivos sino también inferenciales, se aplicó la prueba $t$ para muestras independientes, tomando las proporciones totales observadas como valores promedios (las proporciones totales fueron calculadas, como se verá en los resultados, en base a la sumatoria del valor de centralidad resultante tanto de las palabras relacionadas al paradigma hedónico como al eudaimónico, dividido por el total de valor de centralidad alcanzado por todas las palabras definidoras), y calculando la desviación estándar como la raíz de (p*1-p)/n (Bologna, 2012). Se optó por esta opción dado que si directamente se comparaban las proporciones el $n$ a considerar en los respectivos grupos consistiría en el total del valor de centralidad obtenido por el conjunto de las 15 palabras más centrales, el cual supera los valores de 1000 en ambos grupos. Para este tamaño de $n$ es de esperar que cualquier diferencia en las proporciones sea considerada significativa. En cambio, con el análisis aplicado se tomó como $n$ el total de palabras consideradas, siendo 15 para cada uno de los grupos. Para estos análisis finales se aplicó el software Epidat 3.1.

\section{Resultados}

\section{Concepción de la felicidad en la muestra total}

A continuación se muestran las palabras definidoras de la felicidad con mayores niveles de centralidad (Tabla 1 y Figura 1).

Si se considera el porcentaje en función del puntaje total alcanzado por las 15 primeras palabras, puede apreciarse que el $36.28 \%$ del valor de centralidad total corresponde a definidoras hedónicas de la felicidad, mientras que el $63.72 \%$ restante corresponde a concepciones eudaimónicas de la felicidad. Cabe mencionar además que las tres primeras palabras definidoras de la felicidad, esto es, cuyos valores de centralidad son superiores, corresponden con una 


\section{Tabla 1}

Las 15 palabras definidoras de la felicidad con mayor centralidad. Grupo total $(N=340)$

\begin{tabular}{ccc}
\hline Palabras definidoras & Centralidad & Paradigma \\
\hline amor & 786 & Eudaimónico \\
familia & 738 & Eudaimónico \\
amistad & 679 & Eudaimónico \\
alegría & 466 & Hedónico \\
placer & 441 & Hedónico \\
salud & 379 & Hedónico \\
paz & 360 & Hedónico \\
solidaridad & 271 & Eudaimónico \\
crecimiento personal & 265 & Eudaimónico \\
compañía & 234 & Eudaimónico \\
viajar & 223 & Hedónico \\
trabajo & 213 & Eudaimónico \\
libertad & 208 & Eudaimónico \\
optimismo & 164 & Eudaimónico \\
recreación & 157 & Hedónico \\
\hline
\end{tabular}

Nota: A cada palabra definidora de la felicidad se le asignó un paradigma en el cual se encuadraría dicha concepción de felicidad.

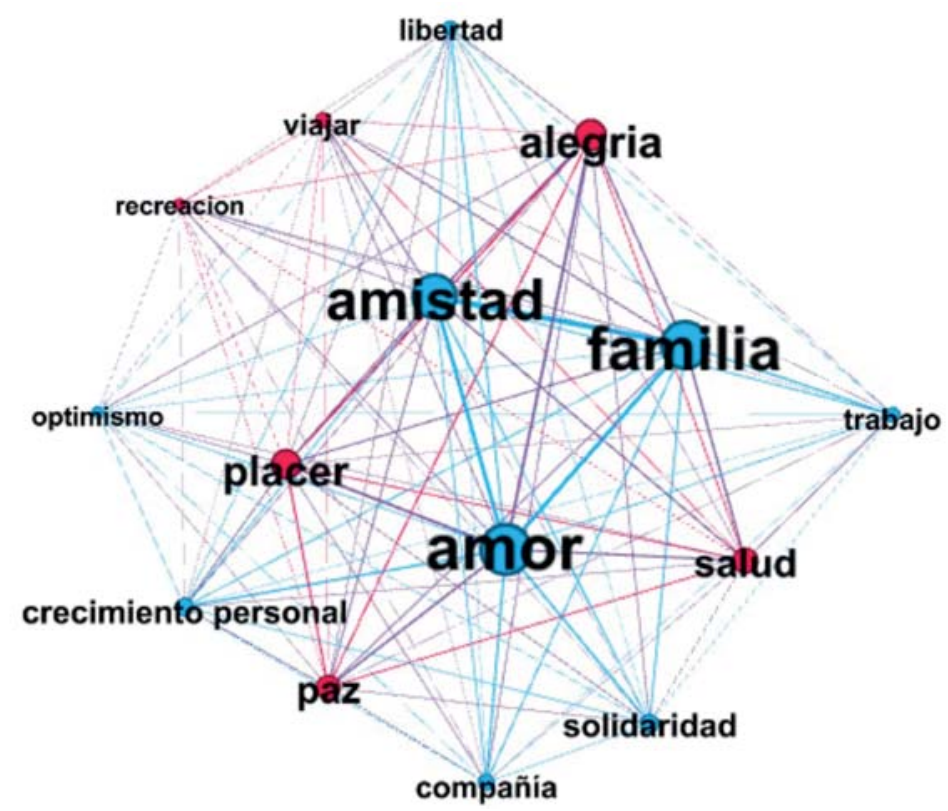

Figura 1. Grafo que muestra la interconexión entre las concepciones de la felicidad que obtuvieron mayores niveles de centralidad ${ }^{1}$.

Con color rojo están representadas las palabras definidoras de la felicidad que corresponderían a un paradigma hedónico, mientras que en celeste están aquellas palabras definidoras de la felicidad correspondientes al paradigma eudaimónico. El tamaño de las etiquetas y los nodos varía en función del nivel de centralidad de los mismos; mientras que el grosor de las aristas da cuenta de una mayor interrelación entre los nodos. 
definición de la felicidad desde un paradigma más eudaimónico.

Concepción de la felicidad en función de los grupos contrastados: Grupo con Felicidad Subjetiva Alta (GFA) vs Grupo con Felicidad Subjetiva Baja (GFB).

En primer lugar se contrasto que los grupos se diferenciaran de una manera estadísticamente significativa en cuanto a los niveles de felicidad evidenciados (Tabla 2).

Como se observa, los grupos difieren de manera estadísticamente significativa en cuanto a los niveles de felicidad subjetiva percibida. Como se esperaba,

Tabla 2

Niveles de felicidad percibida en función de los grupos contrastados

\begin{tabular}{lccccccc}
\hline \multicolumn{9}{c}{ Grupos } \\
\cline { 2 - 8 } & \multicolumn{1}{c}{ GFA $(n=98)$} & GFB $(n=98)$ & & & & \\
& $M$ & $D E$ & $M$ & $D E$ & & & \\
\hline Felicidad & 19.09 & 1.00 & 11.80 & 1.86 & 194 & $34.30 * *$ & 4.93 \\
\hline Nota: ${ }^{* *} p<.001$ & & & & & & &
\end{tabular}

el grupo de felicidad alta (GFA) presenta una media mayor de felicidad $(M=19.09)$ en comparación al grupo de felicidad baja (GFB) quién muestra una media de felicidad menor $(M=11.80)$. Además, es importante mencionar que el tamaño del efecto de estas diferencias es muy grande $(d=4.93)$.

A continuación, se muestran las redes resultantes en cada uno de los grupos comparados (Tabla 3, Figura 2, Tabla 4 y Figura 3).

De manera complementaria, y para poder comparar con un puntaje estándar las diferencias en los valores de centralidad obtenidos por las respectivas palabras en cada uno de los grupos, se muestran de manera gráfica cómo variaron tanto los porcentajes de las palabras individuales (Figura 4 y 5) coincidentes, como los porcentajes totales (sumatoria de los porcentajes obtenidos por el conjunto de palabras referidas al paradigma hedónico y eudaimónico; Figura 6) en función de los grupos contrastados.

Las dos anteriores figuras permiten ver que para el caso de las palabras individuales correspondientes al paradigma hedónico (Figura 4), en el grupo de Felicidad Baja el 80\% de esas palabras superan en los valores de porcentaje a aquellos observados para esas mismas palabras en el grupo Felicidad Alta. Mientras que, para el caso de las palabras individuales correspondientes al paradigma eudaimónico (Figura 5), en el grupo de Felicidad Alta el $71.43 \%$ de esas palabras superan en los valores de porcentaje a aquellos observados para esas mismas palabras en el grupo Felicidad Baja. 


\section{Tabla 3}

Las 15 palabras definidoras de la felicidad con mayor centralidad. Grupo Felicidad Baja $(n=98)$

\begin{tabular}{cccc}
\hline Palabras Definidoras & Centralidad & $\%$ & Paradigma \\
\hline amor & 193 & 13.50 & Eudaimónico \\
familia & 191 & 13.36 & Eudaimónico \\
amistad & 144 & 10.07 & Eudaimónico \\
placer & 117 & 8.18 & Hedónico \\
paz & 113 & 7.90 & Hedónico \\
alegría & 112 & 7.83 & Hedónico \\
salud & 98 & 6.85 & Hedónico \\
crecimiento personal & 80 & 5.59 & Eudaimónico \\
recreación & 63 & 4.41 & Hedónico \\
trabajo & 60 & 4.20 & Eudaimónico \\
viajar & 60 & 4.20 & Hedónico \\
libertad & 60 & 4.20 & Eudaimónico \\
solidaridad & 49 & 3.43 & Eudaimónico \\
música & 47 & 3.29 & Hedónico \\
optimismo & 43 & 3.01 & Eudaimónico \\
\hline
\end{tabular}

Nota: A cada palabra definidora de la felicidad se le asignó un paradigma en el cual se encuadraría dicha concepción de felicidad. \% = porcentaje obtenido en función del valor de centralidad de cada palabra individual divido por la sumatoria total de centralidad alcanzado por el total de las 15 palabras.

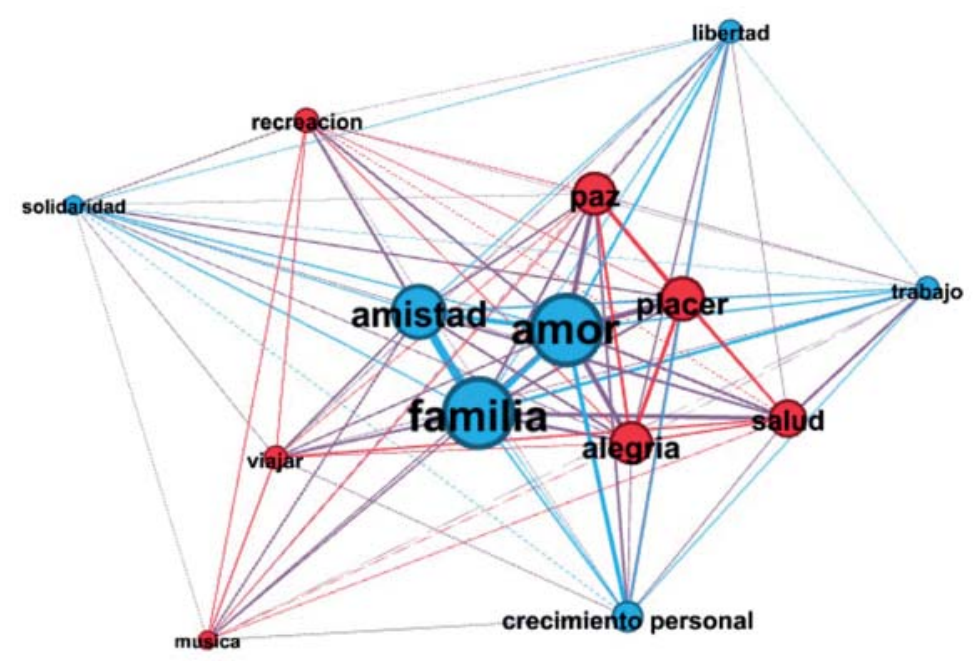

Figura 2. Grafo que muestra la interconexión entre las concepciones de la felicidad que obtuvieron mayores niveles de centralidad. Grupo Felicidad Baja $(n=98){ }^{2}$

2 Con color rojo están representadas las palabras definidoras de la felicidad que corresponderían a un paradigma hedónico, mientras que en celeste están aquellas palabras definidoras de la felicidad correspondientes al paradigma eudaimónico. El tamaño de las etiquetas y los nodos varía en función del nivel de centralidad de los mismos; mientras que el grosor de las aristas da cuenta de una mayor interrelación entre los nodos. 
Tabla 4

Las 15 palabras definidoras de la Felicidad con mayor centralidad. Grupo Felicidad Alta $(n=98)$

\begin{tabular}{cccc}
\hline Palabras Definidoras & Centralidad & $\%$ & Paradigma \\
\hline familia & 257 & 15.33 & Eudaimónico \\
amistad & 247 & 14.74 & Eudaimónico \\
amor & 218 & 13.01 & Eudaimónico \\
alegría & 120 & 7.16 & Hedónico \\
salud & 119 & 7.10 & Hedónico \\
placer & 112 & 6.68 & Hedónico \\
paz & 95 & 5.67 & Hedónico \\
solidaridad & 81 & 4.83 & Eudaimónico \\
compañía & 74 & 4.42 & Eudaimónico \\
trabajo & 72 & 4.30 & Eudaimónico \\
viajar & 62 & 3.70 & Hedónico \\
optimismo & 60 & 3.58 & Eudaimónico \\
crecimiento personal & 60 & 3.58 & Eudaimónico \\
educación & 50 & 2.98 & Eudaimónico \\
compartir & 49 & 2.92 & Eudaimónico \\
\hline
\end{tabular}

Nota: A cada palabra definidora de la felicidad se le asignó un paradigma en el cual se encuadraría dicha concepción de felicidad. $\%=$ porcentaje obtenido en función del valor de centralidad de cada palabra individual divido por la sumatoria total de centralidad alcanzado por el total de las 15 palabras.

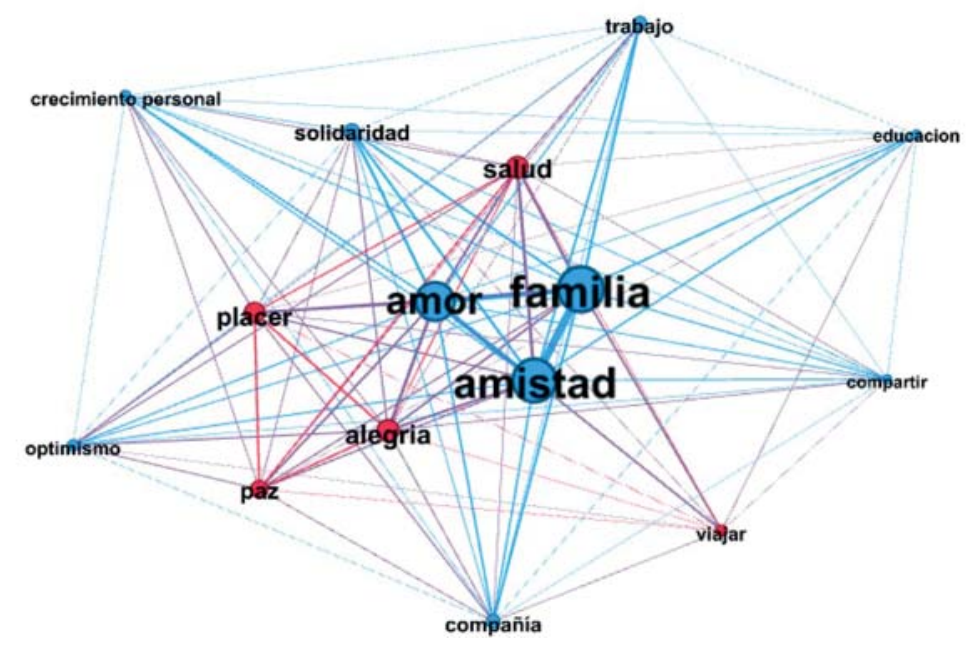

Figura 3. Grafo que muestra la interconexión entre las concepciones de la felicidad que obtuvieron mayores niveles de centralidad. Grupo Felicidad Alta $(n=98) .^{3}$

3 Con color rojo están representadas las palabras definidoras de la felicidad que corresponderían a un paradigma hedónico, mientras que en celeste están aquellas palabras definidoras de la felicidad correspondientes al paradigma eudaimónico. El tamaño de las etiquetas y los nodos varía en función del nivel de centralidad de los mismos; mientras que el grosor de las aristas da cuenta de una mayor interrelación entre los nodos. 


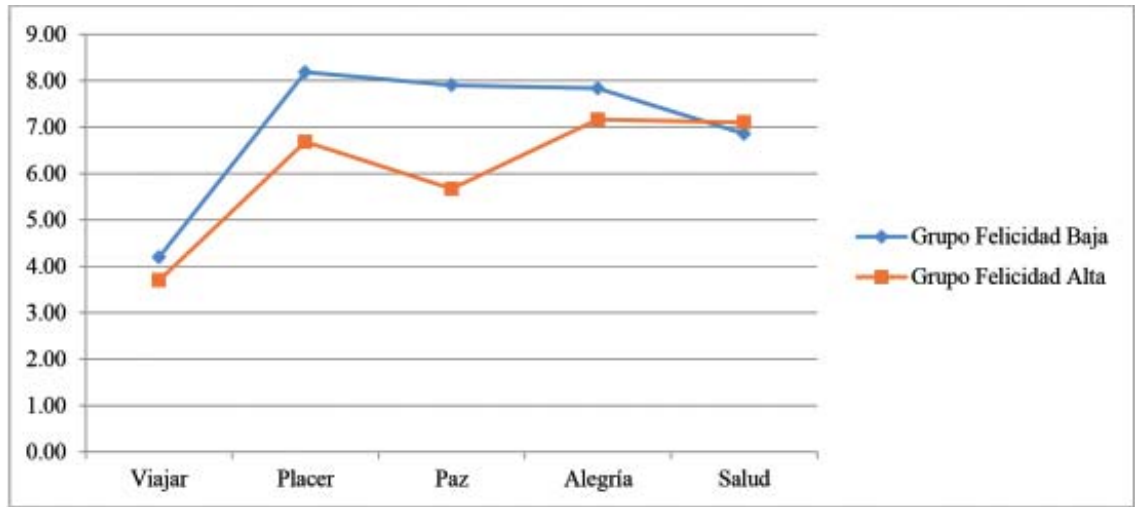

Figura 4. Porcentaje obtenido por las palabras individuales correspondientes al paradigma hedónico en función de los grupos contrastados.

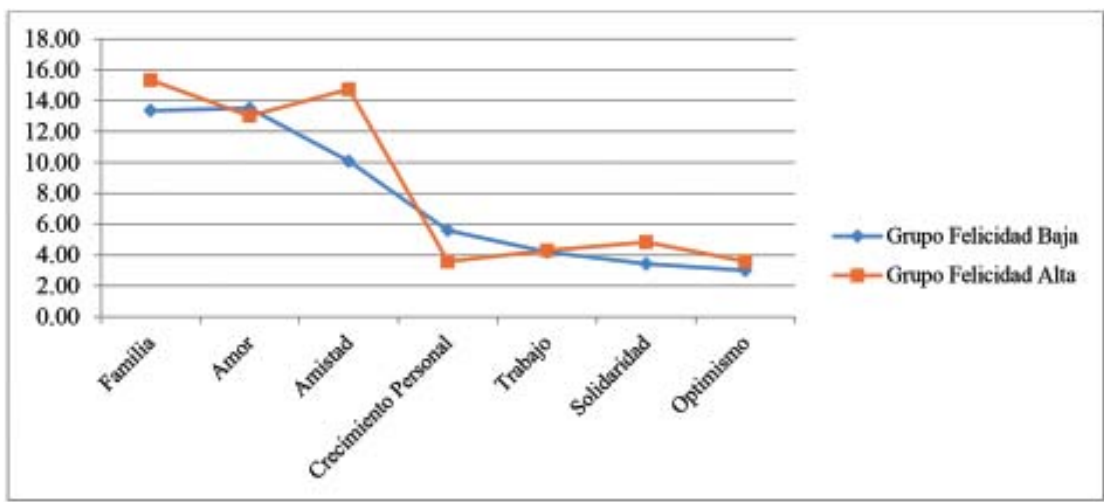

Figura 5. Porcentaje obtenido por las palabras individuales correspondientes al paradigma eudaimónico en función de los grupos contrastados.

Para este último caso (Figura 6), se llevo a cabo una serie de pruebas $t$ para verificar si las proporciones totales observadas, tanto para las concepciones hedónicas como eudaimónicas, variaban de manera estadísticamente significativa en función de los grupos comparados. Al comparar, en primer lugar, entre los grupos si tanto los porcentajes de las concepciones hedónicas como aquellos porcentajes de concepciones eudaimónicas variaban en función de tener mayor o menor felicidad, se observa que los porcentajes varían de forma significativa $(t=2.74, g l=28, p=.01)$. En efecto, puede observarse una diferencia del $12.35 \%$ en ambos casos. Así, mientras en el grupo Baja Felicidad el porcentaje de concepciones hedónicas es del 42.66\%; en el grupo de Alta Felicidad ese porcentaje disminuye al $30.31 \%$. Similarmente, mientras en el grupo Baja Felicidad el porcentaje de concepciones eudaimónicas es del 57.34\%; en el grupo de Alta Felicidad ese porcentaje aumenta al 69.69\%. Luego, al comparar si dentro de los mismos grupos estos porcentajes variaban, se observa que, tanto en el grupo Baja Felicidad $(t=3.26, g l=28, p<.01)$ como en el grupo de Alta Felicidad $(t=8.75, \mathrm{gl}=$ $28, p<.01)$ los porcentajes de las concepciones 


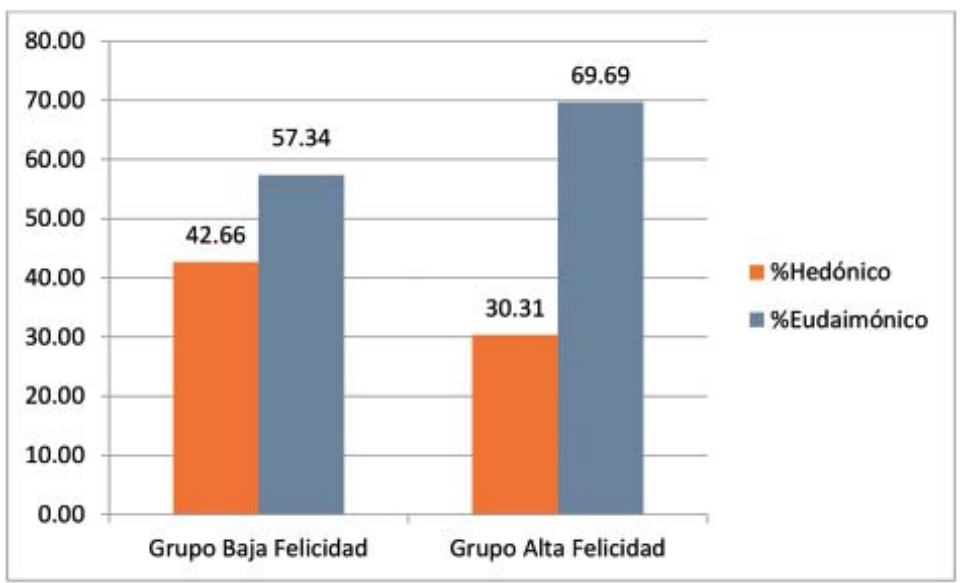

Figura 6. Porcentaje conjunto obtenido por las palabras correspondientes al paradigma hedónico y eudaimónico en función de los grupos contrastados.

eudaimónicas son significativamente más altas que las observadas para las concepciones hedónicas. Sin embargo, las diferencias observadas entre los porcentajes de concepciones hedónicas y eudaimónicas dentro del grupo de Alta Felicidad son más acentuadas (del 39.38\%) que las observadas en el grupo Baja Felicidad (siendo del 14.68\%); en otras palabras, las diferencias entre las concepciones hedónicas y eudaimónicas se acrecientan más del doble en el grupo de Alta Felicidad.

\section{Discusión}

Conocer las concepciones que las personas tienen acerca de la felicidad es una temática que solo recientemente ha comenzado a ser estudiada con mayor énfasis (McMahan \& Renken, 2011). La problemática cobra relevancia dado que las concepciones o paradigmas por medio del cual los sujetos definan su felicidad los lleva a implicarse en diversas acciones o actividades, actividades que contribuirán finalmente a sus niveles de felicidad o bienestar (Bojanowska \& Zalewska, 2015). El presente estudio se propuso contribuir a esta línea de investigación, indagando por un lado qué paradigmas son tenidos en cuenta por los ciudadanos a la hora de definir la felicidad y, por otra parte, verificar si los sujetos varían en sus niveles de felicidad subjetiva en función de cómo hayan definido el concepto de felicidad, ya sea desde un paradigma más hedónico o primordialmente eudaimónico. Dos hipótesis se propusieron en este sentido: 1) los participantes concebirán la felicidad tanto en términos hedónicos como eudaimónicos, y 2) aquellos participantes con mayores niveles de felicidad tendrán una concepción predominantemente eudaimónica de la felicidad.

La primera de las hipótesis pudo corroborarse de manera amplia, dado que las definiciones obtenidas, tanto en la muestra total como en los grupos comparados, contenían tanto componentes hedónicos como eudaimónicos (e.g., Tabla 1 y Figura 1). Esto es coherente con la serie de investigaciones que se han venido desarrollando en los últimos años (Bojanowska \& Zalewska, 2015; Caunt et al., 2013; Denegri et al., 2015; Henderson, Knight, \& Richardson, 2013; Huta \& Ryan, 2009; López-Pérez et al., 2015; McMahan \& Renken 2011; Torner, 2011; Waterman, 1993), y abre la interrogante que muchas de estas investigaciones se han planteado a saber: $i^{e l}$ predominio en las personas de una concepción más eudaimónica o más hedónica de la felicidad tiene 
un impacto en los niveles de bienestar o felicidad percibidos?

En el presente estudio se dió respuesta a este interrogante, aportando evidencia a favor de la segunda hipótesis, esto es que los participantes con mayores niveles de felicidad subjetiva presentan una concepción predominantemente eudaimónica de la felicidad (ver Tabla 3 a Figura 6). Aún más, aquellos individuos con una concepción predominantemente eudaimónica presentaron niveles de felicidad significativamente mayores en comparación con los individuos en donde el paradigma hedónico ocupaba un lugar más central y más equilibrado con respecto a los conceptos eudaimónicos (Tabla 2; Figura 6, diferencia intragrupo: grupo Baja Felicidad). Asimismo, los resultados obtenidos apoyan la tesis de Ryan y Deci (2001) quienes plantean que la forma en que uno defina el bienestar impactará en las actividades que se lleven a cabo en los diversos ámbitos de la vida, acciones que las personas implementarán justamente para lograr el bienestar o felicidad. Además, los datos son coincidentes con los hallazgos de Bojanowska y Zalewska (2015), McMahan y Estes (2011; 2012), y McMahan y Renken (2011).

Haciendo un análisis más molecular de los resultados obtenidos, puede verse que los componentes eudaimónicos mencionados en las definiciones de la felicidad, y que permitieron una mayor distinción entre los grupos (Figura 5), corresponden a conceptos referidos a relaciones positivas (e.g., familia; amistad) y solidaridad. Estos conceptos dados por los participantes son coincidentes con los rasgos propuestos por Riff y Singer (2007) para el logro del potencial humano, principalmente con el propósito en la vida, y las relaciones positivas. Estas definiciones halladas en la investigación también han sido mencionadas por Seligman (2003) como algunos de los rasgos fundamentales para el alcance de la felicidad; así como por McMahan y Renken (2011), quienes propusieron al autodesarrollo y la contribución a los otros como componentes claves de la concepción eudaimónica, y como factores principales en la predicción del bienestar. Sin embargo, y tal como sugiere también Seligman (2003), los resultados no indican que las personas deban renunciar a una concepción hedónica de la felicidad. Por el contrario, las personas más felices de este estudio demostraron tener ambas concepciones, aunque la visión eudaimónica de la felicidad predominaba por sobre la hedónica. En efecto, la distinción más grande evidenciada entre los grupos está con respecto a la valoración dada a los conceptos hedónicos, que fueron muchos menos centrales en la definición de la felicidad si se las compara con los conceptos eudaimónicos concebidos por el grupo con mayor felicidad (Figura 6, diferencia intragrupo: grupo Alta Felicidad). Entre estos conceptos, los que permitieron una mayor distinción entre los grupos fueron (Figura 4) viajar, placer, paz y alegría. A partir de estos resultados es posible hipotetizar también que el alcance de una mayor felicidad se lograría si las personas dedicaran más tiempo a acciones eudaimónicas que a las propiamente hedónicas, pero no si abandonaran por completo las actividades de carácter hedónico.

Finalmente cabe mencionar que el presente estudio fue de carácter ex post facto retrospectivo, es decir, de tipo transversal. Lo anterior indica que no puede garantizarse con el diseño utilizado la antecesión temporal de las variables estudiadas y, por lo tanto, la dirección causal es discutible. En este sentido, sería conveniente que futuras investigaciones propusieran diseños de tipo longitudinal en donde se midiera en una primera fase las concepciones sobre la felicidad y luego, en fases posteriores, los cambios evidenciados en los niveles de felicidad o bienestar percibidos. Un estudio aún más completo podría incluir entre sus mediciones no solo las concepciones que se tenga de la felicidad, sino también las acciones llevadas a cabo en la actividad diaria, acciones que pueden tener un fin más hedónico o eudaimónico. 


\section{Sección de novedad y relevancia}

\section{¿Qué se sabe sobre el tema?}

La búsqueda de la felicidad es uno de los fines más ansiados por los seres humanos. Existen estudios recientes dentro de la psicología que buscan cuáles son los factores involucrados en la felicidad. Contamos con dos grandes enfoques para su estudio, el paradigma hedonista y el paradigma eudaimónico, pero no está clara su relación.

\section{¿Qué añade este artículo?}

Este artículo añade información al concepto de la felicidad con una técnica novedosa como las Redes Semánticas Naturales. Los resultados muestran que la felicidad conlleva tanto componentes hedónicos como eudaimónicos, pero los participantes con mayores niveles de felicidad subjetiva presentan una concepción predominantemente eudaimónica de la felicidad.

\section{Conflicto de intereses}

Los autores manifiestan que no existen conflictos de interés.

\section{Responsabilidad ética}

a) Protección de personas y animales: los autores manifiestan que no fueron realizados experimentos, b) confidencialidad de datos: se siguen protocoles del centro de filiación de los autores, c) derecho a la privacidad y consentimiento informado: en el presente estudio no aparecen datos de participantes.

\section{Referencias}

Aspinwall, L. G., \& Staudinger, U. M. (2007). Psicología del Potencial Humano. España: Gedisa Editorial.

Averill, J. R. (2009). Emotional creativity: Toward «spiritualizing the passions». In S. J. Lopez \& C. R. Snyder (Eds.), The Oxford Handbook of Positive Psychology (pp. 249-259). New York: Oxford University Press.

Baltes, P. B., \& Freund, A. M. (2007). El potencial humano como orquestación de la sabiduría y la optimización selectiva con compensaciones. In L. G. Aspinwall \& U. M. Staudinger (Eds.), Psicología del Potencial Humano (pp. 45-63). España: Gedisa Editorial.

Boehm, J. K., \& Lyubomirsky, S. (2009). The promise of sustainable happiness. In S. J. Lopez \& C. R. Snyder (Eds.), The Oxford Handbook of Positive Psychology (pp. 667-679). New York: Oxford University Press.

Bojanowska, A., \& Zalewska, A. M. (2015). Lay understanding of happiness and the experience of wellbeing: Are some conceptions of happiness more beneficial than others? Journal of Happiness Studies, 17(2), 793-815. doi: 10.1007/s10902-015-9620-1

Bologna, E. (2012). Estadística para Psicología y Educación. Córdoba, Argentina: Brujas.

Borgatti, S. P., Everett, M. G., \& Johnson, J. C. (2013). Analyzing social networks. Los Angeles: SAGE Publications.

Caunt, B. S., Franklin, J., Brodaty, N. E., \& Brodaty, H. (2013). Exploring the causes of subjective wellbeing: A content analysis of peoples' récipes for longterm happiness. Journal of Happiness Studies, 14, 475-499. doi: 10.1007/s10902-012-9339-1

Cohn, M. A., \& Fredrickson, B. L. (2009). Positive emotions. In S. J. Lopez \& C. R. Snyder (Eds.), The Oxford Handbook of Positive Psychology (pp. 1325). New York: Oxford University Press.

Denegri, C. M., García, J. C., \& González, R. N. (2015). Definición de bienestar subjetivo en adultos jóvenes profesionales chilenos. Un estudio con redes semánticas naturales. CES Psicología, 8(1), 77-97. 
Diener, E., Oishi, S., \& Lucas, R. E. (2009). Subjective wellbeing: The science of happiness and life satisfaction. In S. J. Lopez \& C. R. Snyder (Eds.), The Oxford Handbook of Positive Psychology (pp. 187-195). New York: Oxford University Press.

Henderson, L. W., Knight, T., \& Richardson, B. (2013). An exploration of the well-being benefits of hedonic and eudaimonic behaviour. The Journal of Positive Psychology, 8(4), 322-336. doi: 10.1080/17439760. 2013.803596

Huta, V., \& Ryan, R. M. (2009). Pursuing pleasure or virtue: The differential and over lapping well-being benefits of hedonic and eudaimonic motives. Journal of Happiness Studies, 11, 735-762. doi: 10.1007/s1 0902-009-9171-4

Isen, A. M. (2007). El afecto positivo como una fuente de potencial humano. In L. G. Aspinwall \& U. M. Staudinger (Eds.), Psicología del Potencial Humano (pp. 247-269). España: Gedisa Editorial.

Jacomy, M., Heymann, S., Venturini, T., \& Bastian, M. (2011). ForceAtlas2, a graph layout algorithm for handy network visualization. Sciences Po, medialab. Recuperado de https://pdfs.semanticscholar.org/ac53/ ade1902cb209493b541a29e65fab95bee497.pdf

Maddux, J. E. (2009). Stopping the «madness»: Positive psychology and deconstructing the illness ideology and the DSM. In S. J. Lopez \& C. R. Snyder (Eds.), The Oxford Handbook of Positive Psychology (pp. 61-71). New York: Oxford University Press.

McMahan, E. A., \& Estes, D. (2011). Hedonic versus eudaimonic conceptions of well-being: Evidence of differential associations with self-reported wellbeing. Social Indicators Research, 103, 93-108. doi: 10.1007/s11205-010-9698-0

McMahan, E. A., \& Estes, D. (2012). Age-related differences in lay conceptions of well-being and experienced well-being. Journal of Happiness Studies, 13, 79-101. doi: 10.1007/s10902-011-9251-0

McMahan, E. A., \& Renken, M. D. (2011). Eudaimonic conceptions of well-being, meaning in life, and selfreported well-being: Initial test of a mediational model. Personality and Individual Differences, 51, 589-594. doi: 10.1016/j.paid.2011.05.020
Medrano, L. (2013). Medición en Psicología Positiva: desarrollo y validación de instrumentos. Trabajo presentado en la XIV Reunión Nacional y III Encuentro Internacional de la Asociación Argentina de Ciencias del Comportamiento, Córdoba, Argentina.

Montero, I., \& León, O. (2006). A guide for naming research studies in Psychology. International Journal of Clinical and Health Psychology, 7(3), 847-862.

Nakamura, J., \& Csikszentmihalyi, M. (2007). Las fuentes motivadoras de la creatividad contempladas desde el paradigma de la psicología positiva. In L. G. Aspinwall \& U. M. Staudinger (Eds.), Psicología del Potencial Humano (pp. 349-367). España: Gedisa Editorial.

López-Pérez, B., Sánchez, J., \& Gummerum, M. (2015). Children's and adolescents conceptions of happiness. Journal of Happiness Studies, 17(6), 2431-2455. doi: 10.1007/s10902-015-9701-1

Lyubomirsky, S., \& Lepper, H. S. (1999). A measure of subjective happiness: Preliminary reliability and construct validation. Social Indicators Research, 46, 137-155.

Ramírez, E. L., Martínez, G. M., Montemayor, V. P., \& Nieto, C. R. (2009). El análisis del significado humano a través de la técnica de redes semánticas naturales. In J. Vivas (Comp.), Evaluación de Redes Semánticas. Instrumentos y Aplicaciones. Mar del Plata: Eudem.

Riff, C. D., \& Singer, B. (2007) Ironías de la condición humana: Bienestar y salud en camino a la mortalidad. In L. G. Aspinwall \& U. M. Staudinger (Eds.), Psicología del Potencial Humano (pp. 367-389). España: Gedisa Editorial.

Ryan, R. M., \& Deci, E. L. (2001). On happiness and human potentials: A review of research on hedonic and eudaimonic well-being. Annual review of psychology, 52, 141-66.

Seligman, M. E. P. (2003). La Auténtica Felicidad. España: Zeta.

Thalheimer, W., \& Cook, S. (2002). How to calculate effect sizes from published research: A simplified methodology. A Work-Learning Research Publication.

Torner, J. F. (2011). Enduring happiness: Integrating the hedonic and eudaimonicapproaches. The Journal of 
Socio-Economics, 40, 530-537. doi:10.1016/j.so cec.2011.04.003

Valdez, M. J. L. (2002). Las redes semánticas naturales, usos y aplicaciones en psicología social. México: Universidad Autónoma del Estado de México.
Waterman, A. S. (1993). Two conceptions of happiness: Contrasts of personal expressiveness (eudaimonia) and hedonic enjoyment. Journal of Personality and Social Psychology, 64(4), 678-691.

Pablo Ezequiel Flores-Kanter

Universidad Nacional de Córdoba (Argentina)

Licenciado en Psicología

Doctorando, Investigador y Docente. Universidad Nacional de Córdoba, Argentina. Universidad Siglo 21, Córdoba, Argentina Universidad Empresarial Siglo 21, Monseñor Pablo Cabrera Km 8 1², Camino a Pajas Blancas, código postal 5008, Córdoba, Argentina. ORCID: 0000-0002-6712-779X

Autor corresponsal: ezequielfk@gmail.com

Roger Muñoz-Navarro

Universidad de Valencia (España)

Doctor en Psicología

Profesor asociado. Departamento de Personalidad, Evaluación y Tratamientos Psicológicos. Facultad de Psicología. Universidad de Valencia, Valencia, España

Avd. Blasco Ibañez, 21, 46010, Valencia, España

ORCID: 0000-0002-7064-3699

roger.munoz@uv.es

Leonardo Adrián Medrano

Universidad Siglo 21 (Argentina)

Doctor en Psicología

Profesor. Facultad de Psicología. Secretario de Investigación. Universidad Siglo 21, Córdoba, Argentina.

Universidad Empresarial Siglo 21, Monseñor Pablo Cabrera Km 8 1², Camino a Pajas Blancas, código postal 5008, Córdoba, Argentina. ORCID: 0000-0002-3371-5040

Leonardo.Medrano@ues21.edu.ar 